\title{
Second-sound acoustic turbulence in superfluid helium: Decay of the direct and inverse energy cascades
}

\author{
Victor B. Efimov \\ Department of Physics, Lancaster University, Lancaster, LA1 4YB, United Kingdom and \\ Institute of Solid State Physics RAS, Chernogolovka, Russia \\ Andrei N. Ganshin \\ Department of Physics, Lancaster University, Lancaster LA1 4YB, United Kingdom and \\ Laboratory for Elementary-Particle Physics, Cornell University, Ithaca, New York 14853-5001, USA
}

Peter V. E. McClintock ${ }^{*}$

Department of Physics, Lancaster University, Lancaster LA1 4YB, United Kingdom

(Received 26 April 2012; revised manuscript received 26 July 2012; published 23 August 2012)

\begin{abstract}
We have investigated the decay of second-sound acoustic turbulence in superfluid ${ }^{4} \mathrm{He}$ following removal of the driving force that created it. A periodically driven cylindrical resonator of high quality $Q$ factor was used to create one-dimensional second sound of large wave amplitude. The resultant acoustic turbulence involved an energy cascade towards the higher frequencies (smaller scales) where viscous dissipation occurred. Under some conditions, there was also a co-existing inverse energy cascade in the opposite direction, towards lower frequencies, where dissipation also occurred. We have found that the spectral dynamics that occurs when the periodic drive is switched off exhibits complex and interesting features. We show that the wave decay starts from the high-frequency end of the spectrum in both cases, and we have been able to identify and measure a nonlinear decay time related to the interwave interactions, as distinct from the linear decay process due to dissipation. A windowed Fourier analysis reveals that the occurrence of seemingly chaotic oscillations of the individual harmonic amplitudes were observed during the decay.
\end{abstract}

DOI: 10.1103/PhysRevB.86.054515

PACS number(s): 67.25.dt, 47.35.Rs, 47.27.-i, 43.25.+y

\section{INTRODUCTION}

Turbulence may be defined as a far-from-equilibrium state of a nonlinear physical system whose energy distribution extends over many degrees of freedom. Typically, turbulence is characterized by a spectrum in which energy is pumped into the system at one frequency (i.e., on one length scale) and dissipated at a different frequency (length scale). In between lies an inertial frequency interval where there exists a flux of turbulent energy, flowing across the length scales without dissipation.

Richardson noted ${ }^{1}$ that the kinetic energy at first flows in a dissipation-free manner towards successively smaller length scales until it can be dissipated by viscosity. Kinematic turbulence appears in a nonlinear system when kinematic forces predominate over dissipative ones. This will be the case when the (dimensionless) Reynolds number $\operatorname{Re}=v L / v$ is sufficiently large. The movement of fluid past bars or grids, for example, water ${ }^{2}$ or helium, ${ }^{3-7}$ provides typical examples of kinematic turbulence in cases where the relative velocity $v$ of the fluid and the bars is large enough, and/or the kinematic viscosity $v$ is low enough, where $L$ is a characteristic dimension of the system. Liquid helium has an extremely low viscosity, even in its normal (nonsuperfluid) state.

Another well-known paradigm of turbulence, convectively or thermally driven turbulence, arises when the Archimedean forces in the fluid due to a temperature gradient in a gravitational field are sufficient to overcome the effects of viscosity and thermal conduction. ${ }^{8}$ The behavior of the fluid is then characterized by the (dimensionless) Rayleigh number
$\mathrm{Ra}=g \alpha \Delta T L^{3} / \nu \kappa$, where $g$ is the acceleration due to gravity, $\alpha, \nu$, and $\kappa$ are, respectively, the isobaric thermal expansivity, kinematic viscosity, and thermal diffusivity of the fluid, and $\Delta T$ and $L$ are the characteristic temperature difference and the length scale of the flow. Fluid helium is a very convenient medium for the study of convectional turbulence. ${ }^{9}$ Its Rayleigh number at temperatures of $\sim 4-5 \mathrm{~K}$ can be changed over 12 orders of magnitude, while the heat flux undergoes transition from steady linear thermal conductivity to developed turbulence. ${ }^{10}$

One of the most important questions in relation to energy transformation in turbulent processes is the nature of the interaction between excitations of different frequency (or length scale) and, in particular, the time taken for energy transmission between modes.

In this paper we report an experimental investigation of spectral dynamics in acoustic turbulence. This is a widespread phenomenon $^{11-13}$ that manifests in systems as diverse as aurora ${ }^{14}$ terrestrial plasma, ${ }^{15}$ and Bose-condensed atom systems. ${ }^{16}$ It involves the formation of an energy cascade, i.e., a flux of energy from the pumping (injection) frequency to a dissipative spectral range and arises due to the domination of nonlinear effects in the wave propagation over dissipative processes. Here, the dimensionless parameter that determines the behavior of the waves is the acoustic Reynolds number. It is effectively the ratio of the nonlinear wave distortion to dissipation, $\mathrm{Re}_{\mathrm{ac}}=\varepsilon_{\mathrm{NL}} u_{0} \lambda / \pi v_{\mathrm{eff}}$, where $\varepsilon_{\mathrm{NL}}$ is the nonlinear coefficient in the dependence of velocity on wave amplitude, $u_{0}$ is the oscillatory velocity of an element of the medium, $\lambda$ is the wavelength, and $\nu_{\text {eff }}$ is an effective dissipative 
viscosity (a combination of shear, volume viscosity, and thermal conductivity). The condition $\operatorname{Re}_{\mathrm{ac}}<1$ means that any distortions in the wave will disappear, while the condition $\operatorname{Re}_{\mathrm{ac}} \gg 1$ signifies the formation of breakdown in the wave. ${ }^{17}$ The resultant nonlinear distortion of a harmonic wave leads to the formation of a Kolmogorov-like energy cascade from the pumping frequency to the high-frequency dissipative part of the spectrum. ${ }^{18}$ In the particular case of a second-sound wave, the acoustic Reynolds number is defined as $\operatorname{Re}_{\mathrm{ac}} \simeq \alpha_{2} \delta T / \nu$, where $\alpha_{2}$ is the nonlinear coefficient of the second-sound wave velocity, $\delta T$ is the amplitude of the temperature wave, and $v$ is the dissipation coefficient for wave propagation.

For appropriately chosen temperatures, ${ }^{19}$ second-sound waves in superfluid helium are extremely nonlinear. They are also characterized by weak dissipation and by a virtual absence of dispersion (i.e., a linear dispersion relation $\omega_{k}=c_{20} k$ where $c_{20}$ is the wave velocity at vanishingly small amplitude). Intense acoustic waves in media with high nonlinearity, weak dissipation, and a linear dispersion relation provide examples of strong turbulence. ${ }^{20,21}$ second-sound waves thus provide us with an opportunity to study the dynamical processes that occur in strong turbulence.

We observed and reported the direct energy cascade in second-sound acoustic turbulence ${ }^{18}$ in 2006 , noting that it had much in common with phenomena observed earlier ${ }^{22}$ among capillary waves on the surface of liquid hydrogen. Studies of shock waves showed ${ }^{23}$ that, in principle, such energy cascades should exist in $\mathrm{He}$ II at all pressures. Further investigations of the direct cascade ${ }^{24}$ showed that the application of an additional weak low-frequency driving force led to the excitation of combination frequencies and their harmonics through nonlinear wave interactions. The net result was a decrease by a factor of $4 x$ in the energy in the high-frequency domain and a correspondingly faster decrease of the cascade with rising frequency. It was also established that, for higher harmonics of a single driving force, wave motion in the direct cascade is seemingly random and without any phase coherence with the drive $\mathrm{e}^{25}$ - consistent with our perception of these phenomena as being turbulent in character. We then found ${ }^{26}$ that, in addition to the direct cascade where the energy flux is towards higher frequencies, under the right conditions there can also exist an inverse cascade where energy flows in the opposite direction, towards lower frequencies. All of these studies referred to stationary states, but there is also a variety of interesting kinetic phenomena to be considered related, e.g., to the growth and decay of the turbulent state when the driving is switched on or off. The growth processes were shown ${ }^{27}$ to be consistent with a theoretical description based on self-similarity. They also involved the creation of rogue waves ${ }^{28}$ much larger than their neighbors, via nonlinear wave interactions which arguably are closely analogous to the processes responsible for the formation of rogue waves on the ocean. ${ }^{29}$ In what follows we investigate the decay of second-sound wave turbulence, addressing the question of what happens following sudden removal of the driving force that is sustaining steady-state wave turbulence in either the direct or the inverse energy cascade. We will show that there is a characteristic nonlinear decay time and that, like the earlier results from wave turbulence on liquid hydrogen, ${ }^{22}$ the decay starts from the high-frequency end of the spectrum in both cases. A quite unexpected outcome is that, for the inverse cascade only, the amplitudes of the Fourier components undergo seemingly chaotic oscillations during the decay.

We describe the experimental arrangements in Sec. II. In Secs. III and IV we present and discuss our experimental results on the direct and inverse cascades, respectively. We summarize and draw conclusions in Sec. V.

\section{RESONATOR AND EXPERIMENTAL TECHNIQUE}

The experiments were conducted in the high quality cylindrical second-sound resonator previously described. ${ }^{30,31}$ Briefly, a thin-film heater was placed at one end of a quartz cylinder of length $\ell=7 \mathrm{~cm}$ and diameter $D=15 \mathrm{~mm}$. The other end of the resonator was capped by superconducting bolometer, whose transition temperature could be adjusted by application of a magnetic field to maximize its sensitivity. The second-sound temperature oscillation changed the bolometer resistance, which was monitored and recorded on a digital oscilloscope and then transferred to a computer for subsequent analysis. The energy of the second-sound wave is defined as $\varepsilon \simeq \rho C \delta T^{2} / 2 T$; we will discuss the energy flux dynamics of second-sound waves in terms of the square of the wave amplitude $A^{2} \propto \delta T^{2}$.

The quality of the resonator reached $Q \sim 3000-8000$ for the higher resonances $(N>10)$, but was less than this for lower resonances. This increase of $Q$ with rising resonance number may be accounted for in the following way. The normal-fluid/superfluid counterflow in the resonator involves viscous dissipation between the normal-fluid component and the resonator wall, with a penetration depth of $\Lambda=\sqrt{2 \eta / \omega \rho_{n}}$, where $\eta$ is the dynamic viscosity and $\rho_{n}$ is the density of the normal component. Energy loss is caused by wave motion along the wall within the layer $\Lambda$ at distance $L$, which is designated by the interference condition. The "mechanical" quality of the resonator is determined by the parallelism of its reflecting end plates. In our case nonparallelism was $\Delta \ell / \ell \simeq 5 \times 10^{-4}$. The wave in the resonator disappears when the number of reflections $N$ multiplied by $\Delta \ell$ is of the order of the wavelength $\lambda=c / f=2 \pi c / \omega \simeq \Delta \ell \times N$, where $c$ is the second-sound velocity. The wave in this case runs a distance

$$
L=\ell N=\frac{2 \pi c}{\Delta \ell \omega} \times \ell .
$$

The quality of resonator

$$
Q \propto \frac{1}{L \Lambda} \propto \omega^{3 / 2} .
$$

Similar dependencies exist for thermal losses along the resonator surface, losses through defects in the reflecting surfaces, holes in the resonator wall, and its roughness, imperfection of the heater and so on, and have a deterministic character. Estimations of resonator second-sound wave losses in bulk, through surface dissipation by the viscous normal-fluid component, and due to thermal transmission into the wall were published long ago. ${ }^{32}$

Resonator behavior can sometimes be affected by other factors, leading to drastic differences between the $Q$ values of neighboring resonances, but $Q(f)$ is typically monotonic. The 


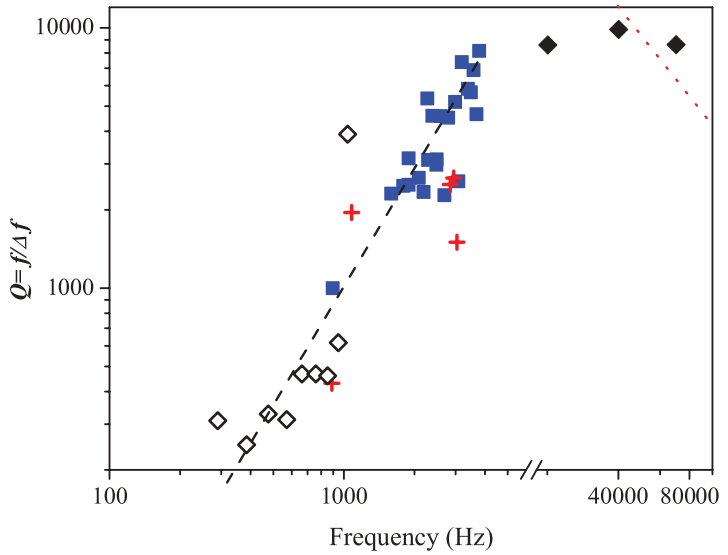

FIG. 1. (Color online) Dependence on frequency $f$ of the resonator quality $Q$ factor at $T=2.08 \mathrm{~K}$ with low excitation. The lower (black) dashed line corresponds to $Q \propto f^{3 / 2}$, and the different symbols represent the results of different experiments. The upper (red) dotted line indicates the calculated resonator $Q$ if it were governed solely by bulk dissipation of the second-sound waves.

experimentally measured dependence of $Q$ on the resonant frequency $f$ is shown in Fig. 1. The $Q \propto \omega^{3 / 2}$ dependence would break down for a wavelength of order $\Delta \ell$, at frequencies $\sim 10^{5} \mathrm{~Hz}$. In our case, however, once the resonator $Q$ has attained 8000-10 000 it becomes only weakly dependent on the resonance number, which occurs at frequencies of $20-40 \mathrm{kHz}$. This indicates the existence of some additional sources of energy loss from the resonator, and it is these that limit the resonator quality $Q$ at high frequencies.

The penetration depth is approximately $2.5 \mu \mathrm{m}$ for a resonant frequency $\sim 1000 \mathrm{~Hz}$ under the conditions of Fig. 1. We suppose that the layer of helium within $\Lambda$ of the wall tends to resist the wave motion. The absolute value of resonator quality should therefore be of order $\pi D^{2} / 4(\pi \times D \times \Lambda) \sim$ 1000. This estimate is very close to the result obtained experimentally.

Bulk second-sound attenuation is of course always present, and is the intrinsic dissipation mechanism usually considered in theoretical analyses. Let us estimate the bulk dissipation within the resonator. Measurements of second-sound bulk attenuation have been reported for temperatures in the range between $0.85 \mathrm{~K}$ and $T_{\lambda}{ }^{33-35}$ The attenuation of a wave of amplitude $A$ as it propagates in the $x$ direction is described by

$$
A(x)=A_{0} e^{-\gamma x} .
$$

The coefficient $\gamma$ depends on temperature and frequency as $\frac{\gamma}{\omega^{2}} \sim \operatorname{const}(T)$. In our experimental temperature range $(1.5<$ $T<2.1 \mathrm{~K})$ the value of $\frac{\gamma}{\omega^{2}}$ was $\sim 10^{-13} \mathrm{~s}^{2} / \mathrm{cm}$. The quality of the resonator just arising from bulk dissipation may be estimated from the ratio ${ }^{35}$

$$
\frac{\gamma}{\omega^{2}}=\frac{\Delta f / f^{2}}{4 \pi u_{2}} \sim 10^{-13}
$$

leading to

$$
Q=\frac{f}{\Delta f} \sim \frac{10^{13}}{4 \pi f u_{2}}
$$

so that, for a frequency $f=1 \mathrm{kHz}, Q \simeq 4 \times 10^{5}$. Thus the bulk dissipation of the second-sound wave becomes dominant at frequencies above $100 \mathrm{kHz}$.

Note that the heat fluxes used ${ }^{30,31}$ were below the threshold of $\sim 20 \mathrm{~mW} \mathrm{~cm}{ }^{-2}$ (Ref. 36) needed to create quantum turbulence ${ }^{37}$ remanent vortices will probably have been present, albeit at too low a density ${ }^{38}$ to cause measurable dissipation.

\section{FREE DECAY OF DIRECT WAVE TURBULENT CASCADE}

Application to the heater of a harmonic signal $\sin (\omega t)$ from a generator launches a temperature (second-sound) wave of double the frequency $\left[A=A_{0} \sin (2 \omega t)\right]$. Under resonance conditions, the excitation leads to a standing wave of amplitude proportional to the quality $Q$ factor of the cavity. For a traveling wave, the strong nonlinearity of second sound steepens the leading edge or creates a tail on the trailing edge of a propagating pulse, depending on the sign of the nonlinearity, which in turn depends on the temperature of the superfluid; in a resonant cavity the corresponding result is multiple harmonics, leading to the direct energy cascade. This means that, for a sufficient excitation amplitude, there is a flux of energy from the pumping frequency towards the high-frequency edge of the spectrum. ${ }^{18,27}$ To study decay of the direct cascade, we excited the system at relatively low resonance numbers (up to the 30th).

Under these conditions, the quality $Q$ factor of the resonator is determined by the energy losses resulting from (a) bulk dissipation and physical imperfections of the resonator (linear losses) and (b) the energy flux into higher harmonics (nonlinear losses). The former is dominant at low excitation levels, while the latter becomes important for stronger excitation. Figure 2(a) shows resonance curves obtained by sweeping the exciting frequency through the 7 th resonance. The $Q$ of the resonator was defined in terms of the width of the resonance curve at a height of $A_{\max } / \sqrt{2}$ where $A_{\max }$ is its peak value. The nonlinear distortion of the wave shape, i.e., the corresponding leakage of energy into higher harmonics, reduces $Q$ at high excitation levels. In the latter case, Fourier analysis of the resonance signal reveals numerous harmonics.

It is to be expected that measurements of the free decay of the standing wave in the resonator should enable one to separate the linear and nonlinear processes. At lower excitation levels, the wave does not generate any energy flux into the higher harmonics, so only the linear process should be seen. For stronger excitation, the energy loss at the moment when the excitation is switched off is determined both by linear processes and also by losses due to the energy flux into higher harmonics.

Figure 2(b) shows the decay of the wave amplitude at the excitation frequency after switchoff, for two different initial excitation levels. It is evident that, for relatively the high initial excitation shown by the (red) triangles, there is indeed an initial faster decay rate. Energy transformation into multiple harmonics ceases when the wave amplitude falls below the value indicated by the dashed line. The time constant of the decay then changes to become equal to the decay constant for lower initial excitation shown by the (blue) circles. 

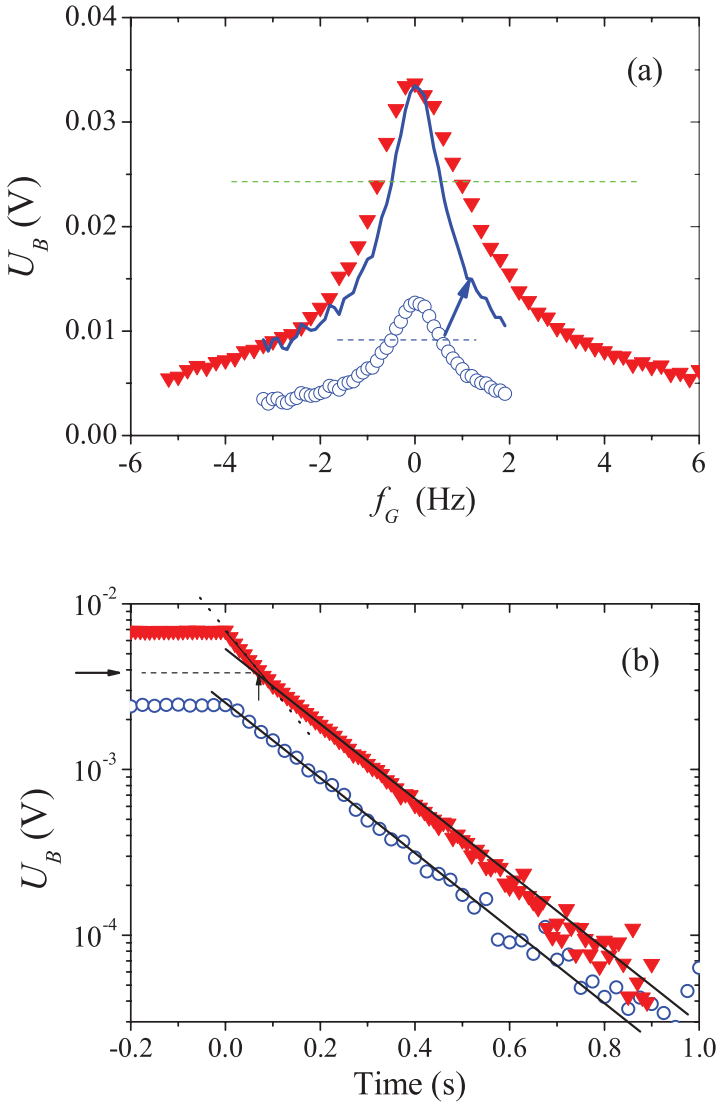

FIG. 2. (Color online) (a) Resonance curves obtained by slowly sweeping the frequency detuning $f_{G}$ of the harmonic driving force near the 7 th resonance at $T=2.08 \mathrm{~K}$. The stationary standing-wave response amplitude $U_{B}$ is plotted for two different fixed driving amplitudes: $U_{G}=4 \mathrm{~V}$ shown by the (red) triangles, and $U_{G}=2 \mathrm{~V}$ shown by the (blue) circles. The (blue) line represents a rescaling of the latter response to facilitate comparison of the widths of the two resonances. The horizontal dashed (green and blue) lines indicate levels of $1 / \sqrt{2}$ of the peak value, to provide indicators of the quality $Q$ factor. (b) Time dependence of the amplitudes of the second-sound signals after cessation of external excitation $U_{G}$ of resonator. The (black) lines represent least-squares fits to the data in each case.

Upon cessation of external excitation, the oscillations in a dissipative system will immediately start decaying with a dissipative eigenfrequency $\omega_{d}=\sqrt{\omega_{0}^{2}-\gamma^{2}}$, where $\omega_{0}$ is the circular frequency corresponding to free vibration of the oscillator in the absence of dissipation and $\gamma$ is the coefficient of dissipation. So the form of the decay should be like

$$
A(t)=A_{0} e^{-\gamma t} \sin \left(\omega t+\varphi_{0}\right),
$$

where $A_{0}$ is the initial value of the oscillation amplitude $A$. The characteristic time of free decay $\tau=1 / \gamma$ is defined by the quality of the resonator and the frequency, as $\tau=\frac{2 Q}{\omega}$. At the 7th resonance in the low excitation limit $Q=240$, which yields a characteristic time $\tau \simeq 0.22 \mathrm{~s}$. This is very close to the experimentally measured value of $\tau \simeq 0.19 \mathrm{~s}$ [Fig. 2(b)]. The process of energy transformation into higher harmonics reduces the characteristic time $\tau$. The initial part of the free decay of signal from $4-\mathrm{V}$ excitation has a faster time dependence ( $\tau_{B} \simeq 0.085 \mathrm{~s}$ ), which then changes to a slower time dependence as the amplitude falls below that indicated by the arrow. Fourier analysis indicates the existence of second, third, and higher harmonics during the initial decay interval.

Energy transformation was measured in more detail on the 11th resonance $\left(f_{G}=516.81\right)$ at $T=2.08 \mathrm{~K}, U=3 \mathrm{~V}(W=$ $10.8 \mathrm{~mW} / \mathrm{cm}^{2}$ ). The quality of the resonator was relatively high in this range $\left(Q=f_{R} / \Delta f \simeq 3900\right)$ so that we were able to observe the decay process over several seconds. The resultant time series was analyzed by use of the windowed Fourier transform method: that is, it was divided into sequential short segments, and an FFT was computed for each of them. The resultant time dependencies of the amplitudes at the fundamental frequency and its harmonics are plotted in Fig. 3.

In this way, it is possible to follow the evolution of more than ten harmonics as the standing wave in the resonator decays. After cessation of pumping, the main harmonic loses energy via the two channels (linear and nonlinear): $\Delta E=\Delta E_{L}+$ $\Delta E_{\mathrm{NL}}$ where $\Delta E$ is the total energy loss per unit time and the $L$ and NL subscripts refer to the linear and nonlinear processes. The latter term appreciably accelerates the decay process at what was the driving frequency. The energy in the higher
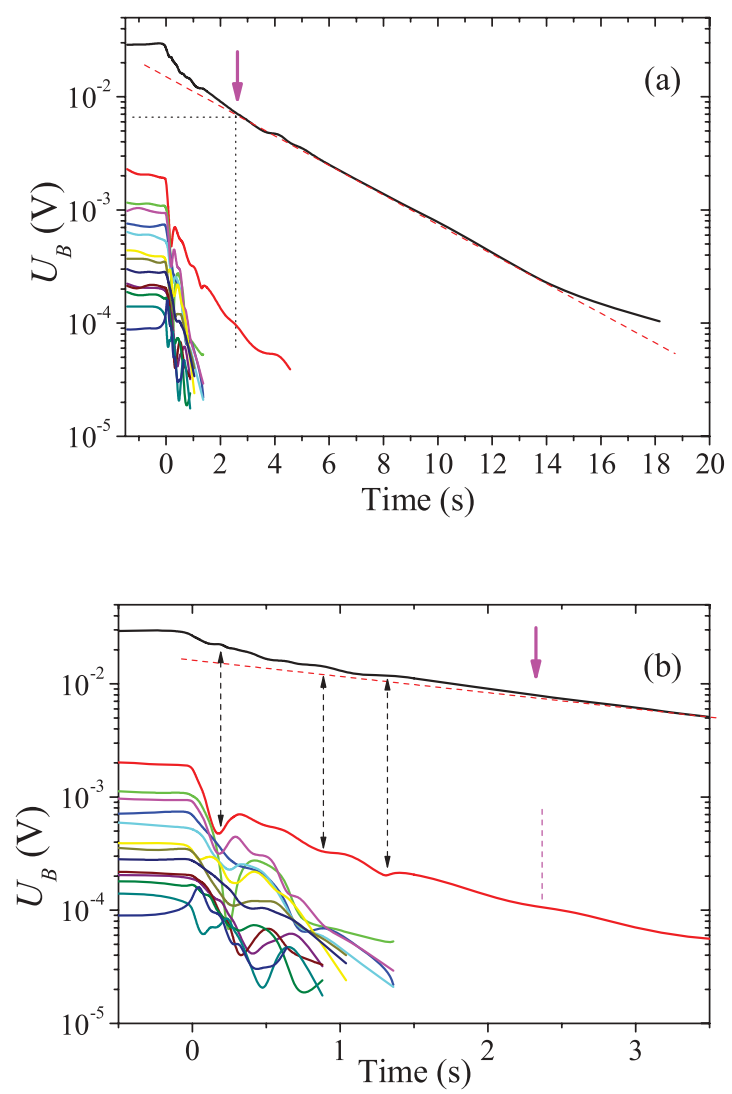

FIG. 3. (Color online) (a) Time dependence of the amplitudes of the harmonics during the decay of the signal, following switching off of the drive at $t=0$ under stationary conditions: 11th resonance, $f_{G}=516.81 \mathrm{~Hz}, T=2.08 \mathrm{~K}$, and $U=3 \mathrm{~V}$ corresponding to $W=$ $10.8 \mathrm{~mW} / \mathrm{cm}^{2}$. The (red) dashed line represents a fit to the linear part of the decaying main harmonic (at the resonant frequency), and the vertical (red) arrow and associated dotted lines indicate the end point of the nonlinear decay. (b) An expansion of the data to show in more detail the chaotic behavior of the higher harmonics just after switching off the driving force. The double-headed arrows indicate correlated maxima/minima at the resonant frequency/second harmonic. 
harmonics, created through the nonlinear interactions during the initial interval, decreases too.

During the initial interval, the redistribution of energy is accompanied by seemingly chaotic amplitude changes among the higher harmonics (starting from the second), as the initially steady-state energy flux collapses. This chaotic behavior is determined by the "inertial" properties of the resonant modes: energy transmission via nonlinear interactions between them is not instantaneous, but requires a finite time. This chaotic behavior continues for $1-1.5 \mathrm{~s}$, which corresponds to $\sim 1500$ periods of the main frequency and thus time for thousands of interactions between the higher harmonics. After this, the amplitudes of the higher harmonics become negligible and any nonlinear interactions between them cease.

The fading away of the higher harmonics begins from the high-frequency end, and then moves towards the main frequency. We note that similar behavior was observed in studies of the free decay of capillary waves on the surface of liquid hydrogen. ${ }^{22}$

During the decay, the rate of nonlinear energy leakage from the main harmonic correlates with the change in amplitude of the second harmonic. Correspondingly, some decreases in amplitude of second harmonic are accompanied by changes in the rate of the free decay of the main harmonic as shown by the arrows in Fig. 3(b).

After $2 \mathrm{~s}$, therefore, the main harmonic decays exponentially with the time constant $\tau_{L}$. The $\tau_{L}$ in Fig. 3 corresponds to a resonator quality $Q \sim 5400$. This value is about $40 \%$ higher than the value determined from the measured quality of the 11 th resonance with an excitation of $U_{G}=2 \mathrm{~V}$. It indicates the existence of nonlinear processes even at this relatively low level of pumping. The linear decay continues for at least $10 \mathrm{~s}$ $\left(\geqslant 10^{4}\right.$ oscillations).

We suggest that the attenuations due to the linear and nonlinear processes are additive, so that we can write the amplitude change in the energy of the main harmonic as

$$
\Delta E_{1}=\Delta E_{L}+\Delta E_{\mathrm{NL}}
$$

The linear time $\tau_{L} \approx 3.3$ s can readily be calculated from the exponential decay of the wave after $t=2 \mathrm{~s}$, when the energy transmission into multiple harmonics has ceased,

$$
A_{L}(t) \approx A \exp \left(-t / \tau_{L}\right)
$$

We can estimate the characteristic nonlinear time from data recorded during the initial interval of free decay. We hypothesize that there is a characteristic nonlinear decay time $\tau_{\mathrm{NL}}$ that remains constant during this interval:

$$
A_{\mathrm{NL}}(t) \approx A_{N L 0} \exp \left(-t / \tau_{\mathrm{NL}}\right)
$$

The difference between $A_{1}^{2}(t)$ and $A_{L}^{2}(t)$ may be identified with the nonlinear energy flux into the direct energy cascade,

$$
A_{\mathrm{NL}}^{2}(t)=A_{1}^{2}(t)-A_{L}^{2}(t) \sim \exp \left(-2 t / \tau_{\mathrm{NL}}\right) .
$$

The difference between $A_{1}^{2}(t)$ and $A_{L}^{2}(t)$ is plotted in Fig. 4. The calculated value of the nonlinear time is about $5 \times$ shorter than the linear time $\left(\tau_{L} \approx 3.3 \mathrm{~s}\right.$ and $\left.\tau_{\mathrm{NL}} \approx 0.6 \mathrm{~s}\right)$.

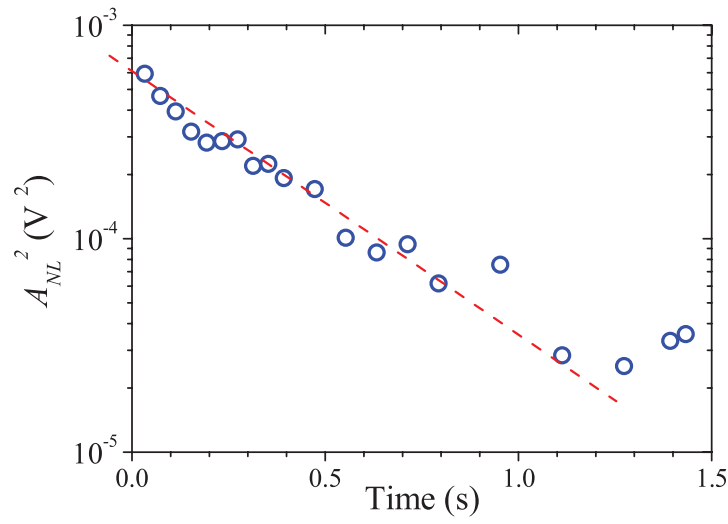

FIG. 4. (Color online) The energy of nonlinear decay, defined as the difference between the energy of the main harmonic and that in the linear (exponential) decay.

\section{DECAY OF THE INVERSE CASCADE}

When the second-sound wave amplitude is large enough, it becomes unstable against decay into subharmonics through processes of the form $\omega_{1} \rightarrow \omega_{2}+\omega_{3}$. This decay instability leads to an inverse energy cascade ${ }^{26}$ (cf. the kinetic instability in turbulent systems ${ }^{39}$ ). Under these conditions, there is a division of the energy flux from the generator at the pumping frequency into components traveling towards higher frequencies (direct cascade) and towards lower frequencies (inverse cascade), respectively. The dissipation of the energy flux in the inverse cascade at the low-frequency end of the spectrum may be attributed to the same processes that cause the resonator $Q$ to become small at low frequencies (see above).

Note that a quite similar parametric process, due to fourwave scattering (modulation instability), is thought to be responsible for the generation of giant "rogue" waves from the background of ordinary wind-driven ocean waves. ${ }^{28,40,41}$ Decay instabilities (especially threshold and near-threshold behavior) have been studied for, e.g., spin waves, ${ }^{42-44}$ magnetohydrodynamic waves in plasma, ${ }^{45}$ and interacting first and second-sound waves in superfluid helium near the superfluid transition. ${ }^{46}$

We have measured the decay of developed inverse energy cascades that include both subharmonics and higher harmonics, under conditions where the driving energy is being shared between the direct and inverse energy cascades. Figure 5(a) illustrates such a free decay following the cessation of external pumping at $t=0$. Figures 5(b)-5(d) plot short samples of the time domain signal extracted at the times indicated by the arrows in Figs. 5(a) and 5(e). The result is a complex decay process during which energy is exchanged between the modes $\omega_{1}+\omega_{2} \Leftrightarrow \omega_{3}$. The main harmonic at $4715 \mathrm{~Hz}$ becomes just one in the set of interacting modes, and the decay looks similar to the seemingly chaotic behavior of the harmonic amplitudes reported above for the decay of the direct cascade. The picture is further complicated by energy transformation between combinational frequencies.

The fading away of the harmonics again starts from the higher frequency end of the spectrum and moves towards the lower frequency spectral range, just as in the case of the direct cascade (see above). A striking feature of Fig. 5(e) is that one of 

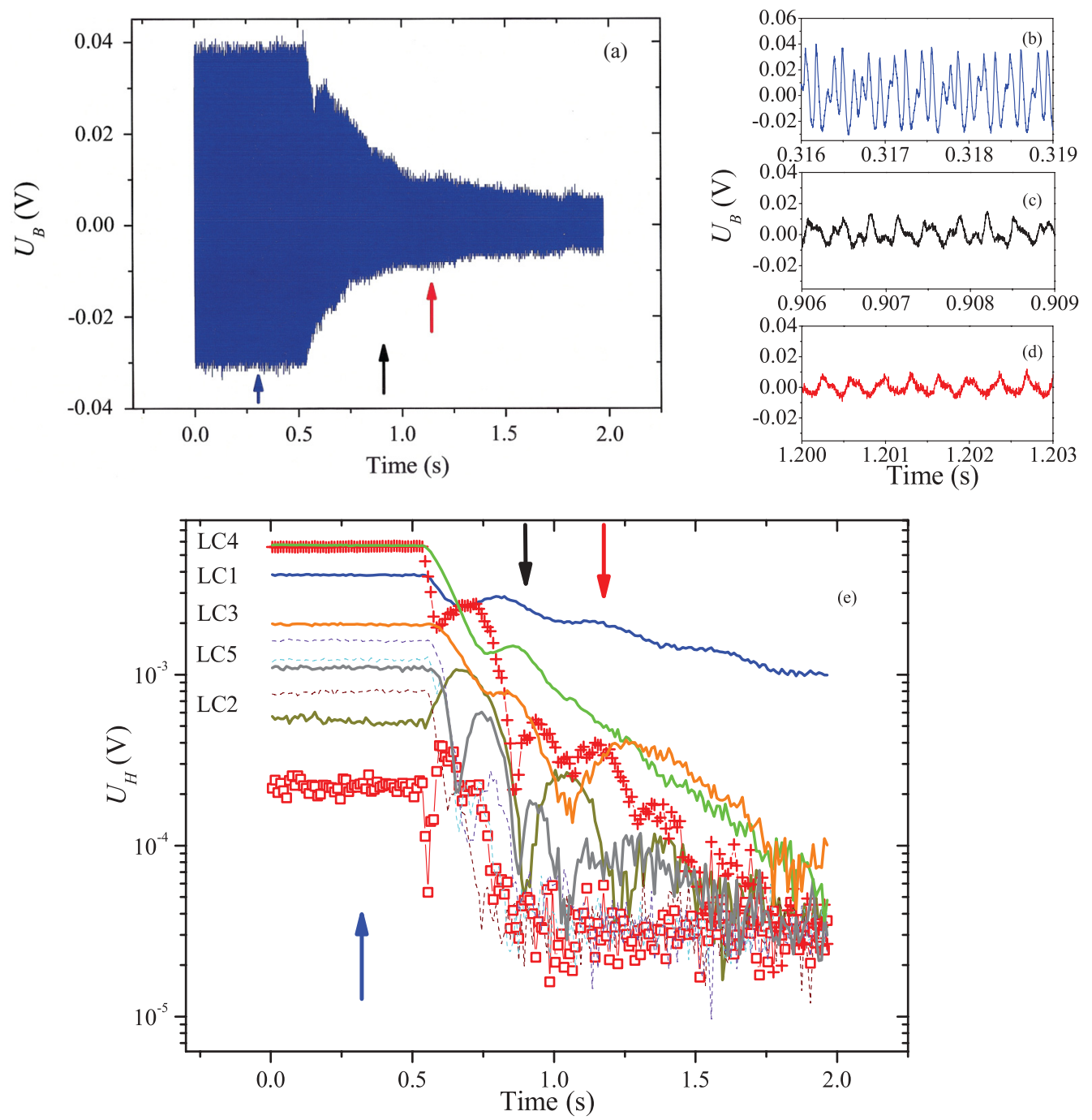

FIG. 5. (Color online) Free decay of the developed inverse cascade following excitation on the 96th resonance at $4715 \mathrm{~Hz}$ with $U_{G}=5 \mathrm{~V}$, $q=30 \mathrm{~mW} \mathrm{~cm}^{-2}$, and $T=2.08 \mathrm{~K}$. (a) Full-length time domain signal $U_{B}(t)$. (b)-(d) Short clips from the time domain signal, taken at the positions indicated by the arrows in (a) from left to right, respectively. (e) Evolution with time of the energy in the different left cascade (LC) subharmonics, higher harmonics, and combinational frequencies, obtained from a windowed Fourier analysis of $U_{B}(t)$ : main harmonic (red) crosses; second harmonic (red) squares. Full lines indicate the behavior of the subharmonics, corresponding to the $\sim 29$ th (LC1), $\sim 38$ th (LC2), $\sim 59$ th (LC3), $\sim 77$ th (LC4), and $\sim 87$ th (LC5) resonances. The combinational frequencies corresponding to interaction between main and LC harmonics are shown as thin dashed lines).

the subharmonics $(f \sim 2850 \mathrm{~Hz})$ persists for much longer than the others. A possible explanation could involve coupling to, and hence energy exchange with, a radial second-sound mode of almost the same frequency. In principle there should be no interaction at all between the longitudinal and radial modes of the resonator; in practice, however, tiny asymmetries and other nonidealities in the construction could conceivably give rise to a finite coupling. Further experimental and theoretical investigations will be needed to settle the question.

\section{CONCLUSIONS}

Our experiments on the decay of second-sound acoustic turbulence following the cessation of pumping have led to some interesting and significant results, in particular as follows:
(1) We have been able to estimate the characteristic nonlinear interaction time. ${ }^{47,48}$ We have shown that, at the temperature used in the present experiments, it is $5 \times$ shorter than the linear decay time related to the small-amplitude $Q$ of the resonator.

(2) For both the direct and inverse cascades, the energy decay starts from the high-frequency end of the spectrum, as observed earlier for capillary waves in liquid hydrogen..$^{22}$ This result illuminates the nature of the quasistationary state with steady pumping and it confirms the reality of an energy flux through a nondissipative inertial range of scales/frequencies.

(3) The discontinuous switching off of the energy pumping leads to seemingly chaotic variations in the harmonic amplitudes as they decrease.

Although the general experimental and theoretical picture is clear, there are a number of details that still require further 
theoretical interpretation, e.g., the oscillatory and seemingly chaotic character of the decaying harmonics.

\section{ACKNOWLEDGMENTS}

We are grateful to G. V. Kolmakov, E. A. Kuznetsov, V. V. Lebedev, A. A. Levchenko, and L. P. Mezhov-Deglin for valuable discussions. The investigations were supported by the Presidium of the Russian Academy of Sciences in framework of the programs "Fundamental Problems of Nonlinear Dynamics," the project NOC FANI in the frame of federal program "Scientific and educational of innovation personnel of Russia," by the Royal Society of London, and by the Engineering and Physical Sciences Research Council (UK). *p.v.e.mcclintock@lancaster.ac.uk

${ }^{1}$ L. F. Richardson, Weather Prediction by Numerical Process (Cambridge University Press, Cambridge, England, 1922).

${ }^{2}$ I. P. D. de Silva and H. J. S. Fernando, Phys. Fluids 6, 2455 (1994).

${ }^{3}$ J. Maurer and P. Tabeling, Europhys. Lett. 43, 29 (1998).

${ }^{4}$ S. R. Stalp, L. Skrbek, and R. J. Donnelly, Phys. Rev. Lett. 82, 4831 (1999).

${ }^{5}$ J. J. Niemela, K. R. Sreenivasan, and R. J. Donnelly, J. Low Temp. Phys. 138, 537 (2005).

${ }^{6}$ D. Charalambous, L. Skrbek, P. C. Hendry, P. V. E. McClintock, and W. F. Vinen, Phys. Rev. E 74, 036307 (2006).

${ }^{7}$ V. B. Efimov, D. Garg, M. Giltrow, P. V. E. McClintock, L. Skrbek, and W. F. Vinen, J. Low Temp. Phys. 158, 462 (2010).

${ }^{8}$ F. H. Busse, Rep. Prog. Phys. 41, 1929 (1978).

${ }^{9}$ J. J. Niemela and K. R. Sreenivasan, J. Low Temp. Phys. 143, 163 (2006).

${ }^{10}$ J. J. Niemela, L. Skrbek, K. R. Sreenivasan, and R. J. Donnelly, Nature (London) 404, 837 (2000).

${ }^{11}$ V. E. Zakharov and R. Z. Sagdeev, Doklady Akad. Nauk USSR 192, 297 (1970)

${ }^{12}$ G. E. Falkovich and A. V. Shafarenko, J. Nonlinear Sci. 1, 457 (1995).

${ }^{13}$ V. S. Lvov, Y. Lvov, A. C. Newell, and V. Zakharov, Phys. Rev. E 56, 390 (1997).

${ }^{14}$ J. E. Wahlund, P. Louarn, T. Chust, H. Deferaudy, A. Roux, B. Holback, P. O. Dovner, and G. Holmgren, Geophys. Res. Lett. 21, 1831 (1994).

${ }^{15}$ V. F. Virko, G. S. Kirichenko, and K. P. Shamrai, Plasma Sources Sci. Technol. 12, 217 (2003).

${ }^{16}$ S. Khlebnikov, Phys. Rev. A 66, 063606 (2002).

${ }^{17}$ V. B. Efimov, G. V. Kolmakov, A. S. Kuliev, and L. P. MezhovDeglin, Low Temp. Phys. 24, 81 (1998).

${ }^{18}$ G. V. Kolmakov, V. B. Efimov, A. N. Ganshin, P. V. E. McClintock, and L. P. Mezhov-Deglin, Phys. Rev. Lett. 97, 155301 (2006).

${ }^{19}$ A. J. Dessler and W. H. Fairbank, Phys. Rev. 104, 6 (1956).

${ }^{20}$ B. B. Kadomtsev and V. I. Karpman, Sov. Phys. Usp. 14, 40 (1971).

${ }^{21}$ S. N. Gurbatov, A. I. Saichev, and I. G. Yakushkin, Usp. Fiz. Nauk 141, 221 (1983).

${ }^{22}$ G. V. Kolmakov, A. A. Levchenko, M. Y. Brazhnikov, L. P. MezhovDeglin, A. N. Silchenko, and P. V. E. McClintock, Phys. Rev. Lett. 93, 074501 (2004).

${ }^{23}$ G. V. Kolmakov, V. B. Efimov, A. N. Ganshin, P. V. E. McClintock, E. V. Lebedeva, and L. P. Mezhov-Deglin, Low Temp. Phys. 32, 999 (2006).
${ }^{24}$ P. V. E. McCintock, V. B. Efimov, A. N. Ganshin, G. V. Kolmakov, and L. P. Mezhov-Deglin, J. Low Temp. Phys. 150, 394 (2008).

${ }^{25}$ V. B. Efimov, A. N. Ganshin, and P. V. E. McClintock, Phys. Rev. E 78, 066611 (2008).

${ }^{26}$ A. N. Ganshin, V. B. Efimov, G. V. Kolmakov, L. P. Mezhov-Deglin, and P. V. E. McClintock, Phys. Rev. Lett. 101, 065303 (2008).

${ }^{27}$ A. N. Ganshin, V. B. Efimov, G. V. Kolmakov, L. P. Mezhov-Deglin, and P. V. E. McClintock, New J. Phys. 12, 083047 (2010).

${ }^{28}$ V. B. Efimov, A. N. Ganshin, G. V. Kolmakov, P. V. E. McClintock, and L. P. Mezhov-Deglin, Eur. Phys. J. Special Topics 185, 181 (2010).

${ }^{29}$ V. Ruban, Y. Kodama, M. Ruderman, J. Dudley, R. Grimshaw, P. V. E. McClintock, M. Onorato, C. Kharif, E. Pelinovsky, T. Soomere et al., Eur. Phys. J. Special Topics 185, 5 (2010).

${ }^{30}$ V. B. Efimov, A. N. Ganshin, P. V. E. McClintock, G. V. Kolmakov, and L. P. Mezhov-Deglin, J. Low Temp. Phys. 145, 155 (2006).

${ }^{31}$ V. B. Efimov, A. N. Ganshin, P. V. E. McClintock, G. V. Kolmakov, and L. P. Mezhov-Deglin, J. Low Temp. Phys. 148, 251 (2007).

${ }^{32}$ V. P. Peshkov, Sov. Phys. JETP 18, 857 (1948).

${ }^{33}$ K. R. Atkins and K. H. Hart, Phys. Rev. 92, 204 (1953).

${ }^{34}$ W. B. Hanson and J. R. Pellam, Phys. Rev. 95, 321 (1954).

${ }^{35}$ K. N. Zinov'eva, Sov. Phys. JETP 4, 36 (1957).

${ }^{36}$ S. K. Nemirovskii and A. N. Tsoi, Cryogenics 29, 985 (1989).

${ }^{37}$ W. F. Vinen, J. Low Temp. Phys. 145, 7 (2006).

${ }^{38}$ D. D. Awschalom and K. W. Schwarz, Phys. Rev. Lett. 52, 49 (1984).

${ }^{39}$ V. S. L'vov and V. B. Cherepanov, Sov. Phys. JETP 54, 746 (1982).

${ }^{40}$ A. I. Dyachenko and V. E. Zakharov, JETP Lett. 81, 255 (2005).

${ }^{41}$ M. Onorato, D. Proment, and A. Toffoli, Phys. Rev. Lett. 107, 184502 (2011).

${ }^{42}$ P. W. Anderson and H. Suhl, Phys. Rev. 100, 1788 (1955).

${ }^{43}$ V. S. L'vov, Wave Turbulence under Parametric Excitation. Applications to Magnets (Springer, Berlin, 1994).

${ }^{44}$ T. Matsushita, R. Nomura, H. H. Hensley, H. Shiga, and T. Mizusaki, J. Low Temp. Phys. 105, 67 (1996).

${ }^{45}$ S. R. Spangler, J. A. Leckband, and I. H. Cairns, Phys. Plasmas 4, 846 (1997).

${ }^{46}$ D. Rinberg, V. Cherepanov, and V. Steinberg, Phys. Rev. Lett. 76, 2105 (1996).

${ }^{47}$ O. V. Rudenko, Usp. Fiz. Nauk 165, 1011 (1995).

${ }^{48}$ O. V. Rudenko, C. M. Hedberg, and B. O. Enflo, Acoust. Phys. 47, 452 (2001). 